\title{
Incorporation of Whole Spine Screening in Magnetic Resonance Imaging Protocols for Low Back Pain: A Valuable Addition
}

\author{
Akhilesh Rao ${ }^{1}$, Atul Mishra ${ }^{2}$, Yayati Pimpalwar ${ }^{3}$, Ravinder Sahdev ${ }^{1}$, Neha Yadu $^{1}$ \\ ${ }^{1}$ Department of Radiodiagnosis \& Imaging, Command Hospital (CC), Lucknow, India \\ ${ }^{2}$ Department of Radiodiagnosis \& Imaging, Military Hospital (CTC), Pune, India \\ ${ }^{3}$ Department of Radiodiagnosis \& Imaging, Military Hospital, Ahmedabad, India
}

Study Design: A retrospective review of lumbar magnetic resonance imaging (MRI) studies conducted at the Department of Radiodiagnosis \& Imaging of a Tertiary Care Armed Forces Hospital between May 2014 and May 2016.

Purpose: To assess the advantages of incorporating sagittal screening of the whole spine in protocols for conventional lumbar spine MRI for patients presenting with low back pain

Overview of Literature: Advances in MRI have resulted in faster examinations, particularly for patients with low back pain. The additional detection of incidental abnormalities on MRI helps to improve patient outcomes by providing a swifter definitive diagnosis. Because low back pain is extremely common, any change to the diagnostic and treatment approach has a significant impact on health care resources.

Methods: We documented all additional incidental findings detected on sagittal screenings of the spine that were of clinical significance and would otherwise have been undiagnosed.

Results: A total of 1,837 patients who met our inclusion criteria underwent MRI of the lumbar spine. The mean age of the study population was 45.7 years; $66.8 \%$ were men and $33.2 \%$ women. Approximately $26.7 \%$ of the patients were diagnosed with incidental findings. These included determining the level of indeterminate vertebrae, incidental findings of space-occupying lesions of the cervicothoracic spine, myelomalacic changes, and compression fractures at cervicothoracic levels.

Conclusions: We propose that T2-weighted sagittal screening of the whole spine be included as a routine sequence when imaging the lumbosacral spine for suspected degenerative pathology of the intervertebral discs.

Keywords: Magnetic resonance imaging; Low back pain; Lumbar spine; Compression fracture; Spinal cord screening

\section{Introduction}

Recent advances in the technology of magnetic resonance imaging (MRI) have resulted in faster examinations, including for patients with low back pain. The early use of
MRI in the management and work-up of these patients has been shown to be beneficial. When incidental abnormalities are detected with MRI, this can help to improve patient outcomes by providing a swifter definitive diagnosis and avoiding needless interventions and a delay in

Received Jan 6, 2017; Revised Feb 5, 2017; Accepted Mar 1, 2017

Corresponding author: Akhilesh Rao

Department of Radiodiagnosis \& Imaging, Command Hospital (CC), Lucknow, India

Tel: +91-7054166991, Fax: +91-0802459630, E-mail: raoakhilesh@yahoo.com 
administering the definitive treatment. Because low back pain is extremely common, any change in the diagnostic and treatment approach is certain to have a significant impact on health care resources.

Back pain is a common clinical condition and is a frequent cause of referral for lumbar spine MRI. In addition to the conventional sagittal and axial images of the lumbar spine and the coronal short tau inversion recovery sequence (STIR), sagittal screening of the whole spine can provide diagnostically significant information in a small percentage of patients. Additional information that can be obtained by sagittal screening of whole spine includes determining the level of indeterminate vertebrae, incidental findings of intramedullary, intradural extramedullary, and extradural lesions of the cervicothoracic spine, myelomalacic changes, and compression fractures at the cervicothoracic level that could be causing significant neural compromise.

The aim of this study was to assess the advantages of incorporating sagittal screening of the whole spine in protocols for conventional lumbar spine MRI for patients presenting with low back pain.

\section{Materials and Methods}

This was a retrospective review of the lumbar MRI studies performed at our center, a tertiary care armed forces hospital. Sagittal screening of the whole spine is part of the routine lumbar spine imaging protocol at our department, in addition to the conventional sagittal and axial images of the lumbar spine and the coronal STIR images. The study period was from May 2014 to May 2016.

\section{Participants}

All patients presenting at our department during the study period with complaints of low back pain with or without associated neurological symptoms for lumbar spine MRI were included in this study. There were no exclusion criteria.

This study conformed to the widely accepted ethical principles that guide human-based research.

As this was a retrospective observational study that involves no additional risk to the patients, we received approval for the study from the Institutional Ethics Committee, who waived the requirement to obtain consent.

\section{Technical information}

The patients underwent a lumbar spine MRI examination using a 1.5 Tesla MR system (MAGNETOM Avanto, Siemens Healthcare, Erlangen, Germany). In addition to sequences imaging the lumbar spine, T2-weighted sagittal images of the cervicothoracic spine were acquired using the following parameters: echo time $=98 \mathrm{~ms}$, repetition time $=3,900 \mathrm{~ms}$, slice thickness $=3.3 \mathrm{~mm}$, bandwidth $=198 \mathrm{~Hz} / \mathrm{px}$, field of view $=512 \times 230$, acquisition matrix $=216 \times 320$, and voxel size $=0.7 \mathrm{~mm} \times 0.7 \mathrm{~mm} \times 3 \mathrm{~mm}$; no interpolation was applied. The total acquisition time was 65 seconds.

Post-processing software (syngo Workspace, Siemens) was used to fuse the cervicothoracic and lumbosacral sequences to create a single $\mathrm{T} 2$-weighted sagittal sequence of the entire spine.

\section{Image and data analysis}

All the lumbar spine MRI studies performed at our center during the study period were retrospectively reviewed. Any additional findings detected on the sagittal screening of the spine, which had clinical significance and would otherwise have been undiagnosed, were documented. These were classified into the following categories: (1) Lumbosacral transitional vertebrae (LSTV), (2) Atlantoaxial dislocation (AAD), (3) Intramedullary, intradural extramedullary, and extradural lesions of the cervicothoracic spine, (4) Myelomalacic changes in the cervicothoracic spine, (5) Old healed fractures causing significant spinal canal compromise in cervical and thoracic vertebrae.

\section{Results}

A total of 1,837 patients who met our inclusion criteria underwent MRI of the lumbosacral spine. These comprised 1227 men (66.8\%) and 610 women (33.2\%), with a mean age of 45.7 years (range, 16 to 86 years). The age distribution was as follows: $\leq 29$ years, 202 patients (11\%); $30-49$ years, 863 patients (47\%); 50-69 years, 477 patients (26\%); and $\geq 70$ years, 293 patients (16\%).

Of the 1,837 patients imaged, 489 (26.7\%) were incidentally diagnosed with the conditions listed earlier (Fig. 1). These comprised 336 men (18.3\%) and 86 women (4.6\%). In addition, 671 patients (36.5\%) were incidentally diag- 
nosed with imaging features of cervical spondylosis; however, because there was no associated neural involvement, these findings were of doubtful clinical significance.

LSTV was the most common finding from our five categories, with a prevalence of about $22.9 \%(n=422)$. More of our patients had sacralized lumbar vertebra (LV) 5 vertebrae $(n=398)$ than lumbarized SV1 vertebrae $(n=24)$.

AAD was detected in $0.010 \%$ of the patients $(n=18)$. Most of these patients were aged 20-29 years $(n=6), 30-39$ years $(n=5)$, or $40-49$ years $(n=2)$. None of these patients exhibited symptoms of AAD.

Space-occupying lesions in the cervical and thoracic cord were detected in $0.007 \%$ of the patients $(n=13)$, eight men and five women. Of these, we found seven lesions in the thoracic spinal cord and six in the cervical cord. The anatomical distribution of these lesions into intramedullary, intradural extramedullary, and extradural locations is shown in Table 1. The intramedullary lesion in the thoracic spinal cord was later confirmed to be a spinal ependymoma. Of the four intradural extramedullary lesions, three were meningiomas and the fourth was a neurofi-

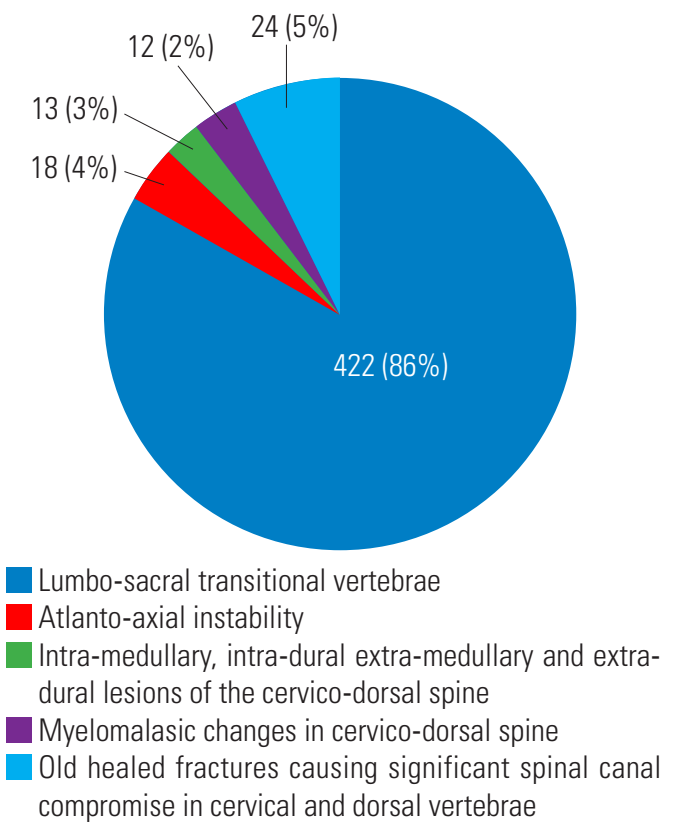

Fig. 1. Pie chart showing the numbers of patients with the incidentally diagnosed lesions. Number of patients incidentally diagnosed. broma.

Myelomalacic changes in the cervical and thoracic spinal cord were detected in $0.006 \%$ of the patients $(n=12)$, eight men and four women. Of these, 11 had myelomalacia in the cervical cord, secondary to herniated intervertebral disk. One patient with an undiagnosed vertebral fracture of the D12 vertebra causing significant compression of the spinal cord was found to have myelomalacic changes in the thoracic cord.

Undiagnosed old healed fractures causing significant spinal canal compromise in cervical and thoracic vertebrae were detected in $0.013 \%$ patients $(n=24)$, three men and 21 women. The women were all in the postmenopausal age group, and the men were aged 60-69 years $(\mathrm{n}=1)$ and $70-79$ years $(\mathrm{n}=2)$.

\section{Discussion}

The discovery of incidental lesions has always been a part of clinical practice and it is worth noting that sometimes an incidental finding can prove even more important than the suspected condition. In this study, the most common incidental finding observed was that of LSTV.

Transitional vertebrae should really be considered as variants rather than actual malformations. The reported prevalence of LSTV in the literature is between $4 \%$ and $36 \%$ [1]. They are related to a shift within the vertical segmentation of the spine that not only involves the osseous structures but also affects the levels of nerves, vessels, muscles, and other anatomic structures [2]. Cranial shift has been reported to be more common than caudal shift, with the traditional numerical distribution of the vertebrae seen in around two-thirds of the population. Transitional vertebrae commonly involve either lumbarization of the first sacral vertebra or sacralization of the fifth lumbar vertebra.

Correct identification of an LSTV is crucial because inaccurate identification may lead to surgical and procedural errors, in addition to poor correlation with clinical symptoms (Fig. 2). There is also the debatable "Bertolotti Syndrome," described in the literature since 1917 and

Table 1. The anatomical distribution of incidentally diagnosed intra-medullary, intra-dural extra-medullary and extra-dural lesions

\begin{tabular}{lccc} 
Regional distribution & Intra-medullary & Intra-dural extra-medullary & Extra-dural \\
Cervical spine & Nil & 3 & 3 \\
Dorsal spine & 1 & 4 & 2 \\
\hline
\end{tabular}




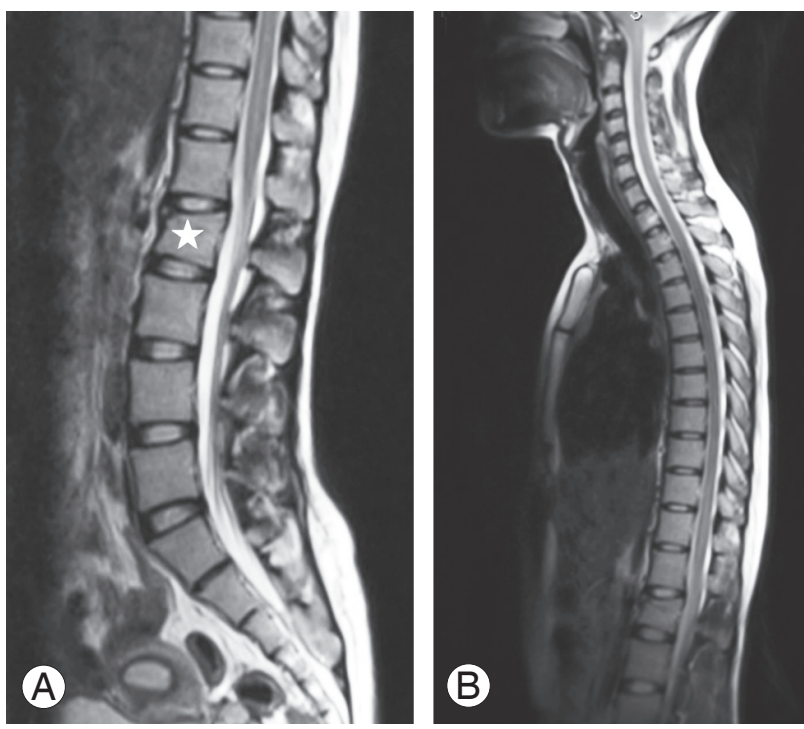

Fig. 2. Lumbar magnetic resonance imaging for a 24-year-old female athlete with a history of trauma and radiographic evidence of a fracture of lumbar vertebra (LV) 2. (A) T2-weighted sagittal image revealing a compression fracture of LV2 (star). (B) A T2-weighted sagittal screening of the whole spine revealed that the patient had a transitional vertebra with a caudal shift and that the affected vertebral level was LV1.

supported by many, which associates low back pain and LSTV [3]. Another potential complication related to the incorrect numbering of the vertebrae is a problem with determining the level for the administration of epidural or intradural anesthetics. Studies [4] have shown that an LSTV can not only affect the position of the conus medullaris but also the intercrestal line (a line connecting the highest points of the iliac crests), which is used as a landmark for needle insertion and usually corresponds with the LV4/LV5 level.

If an LSTV is suspected on imaging, it should be established whether it represents a sacralized LV5 or lumbarized SV1. The true nature of lower vertebral segmentation can sometimes be determined from standard radiographs that include the dorsolumbar junction. However, in current clinical practice, these radiographs are not routinely available when imaging studies of the lumbar spine are being reported. There have been several attempts to number LSTV based on various MRI criteria, including the level of the aortic bifurcation, right renal artery, conus medullaris, and iliolumbar ligament [5]. However, according to Konin and Walz [3], there is no foolproof method for accurately numbering a transitional segment in the absence of highquality imaging of the entirety of the spine. Counting the vertebrae beginning from $\mathrm{C} 2$ is the only definitive method

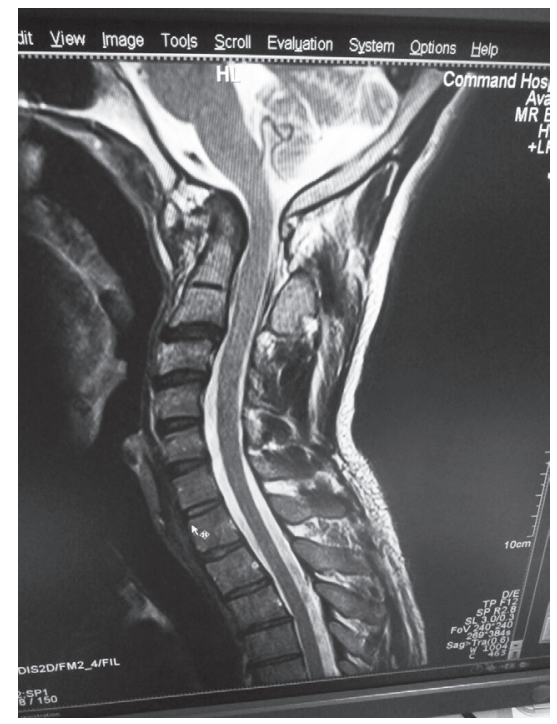

Fig. 3. A T2-weighted sagittal image of the cervical spine of a 42-year-old man with low back pain incidentally revealed atlantoaxial dislocation.

for diagnosing transitional vertebrae.

$\mathrm{AAD}$ is characterized by an increased distance between the atlas (C1) and axis (C2). This is commonly the result of a bony or a ligamentous abnormality. Neurological symptoms occur because of the involvement of the spinal cord or adjacent nerve roots. Studies of AAD have not shown a particular prevalence of the condition in any age group, but there is a higher risk for younger individuals with Down syndrome [6] and older patients with rheumatoid arthritis [7]. Irrespective of the etiology, AAD has a clear potential for neurological compromise (Fig. 3). Of the several different methods for diagnosing AAD, the most important involves the measurement of atlantoaxial joint articulation using the atlantodental interval. This is a measurement of the distance between the posterior aspect of the anterior atlas ring and the anterior aspect of the odontoid process [8]. Screening the whole spine including the cervical region can prove useful in diagnosing AAD even in patients with no overt symptoms, thus aiding the early diagnosis and treatment of these patients.

Spinal cord neoplasms in the early stage usually present with nonspecific symptoms, including local pain and/or stiffness. These early symptoms mimic degenerative disease of the lumbar spine and are occasionally detected incidentally [9]. Screening of the whole spine during imaging of the lumbosacral spine can prove invaluable in this regard. Sagittal MRI sections of the entire spine may be rapidly visualized for the characterization of these lesions 


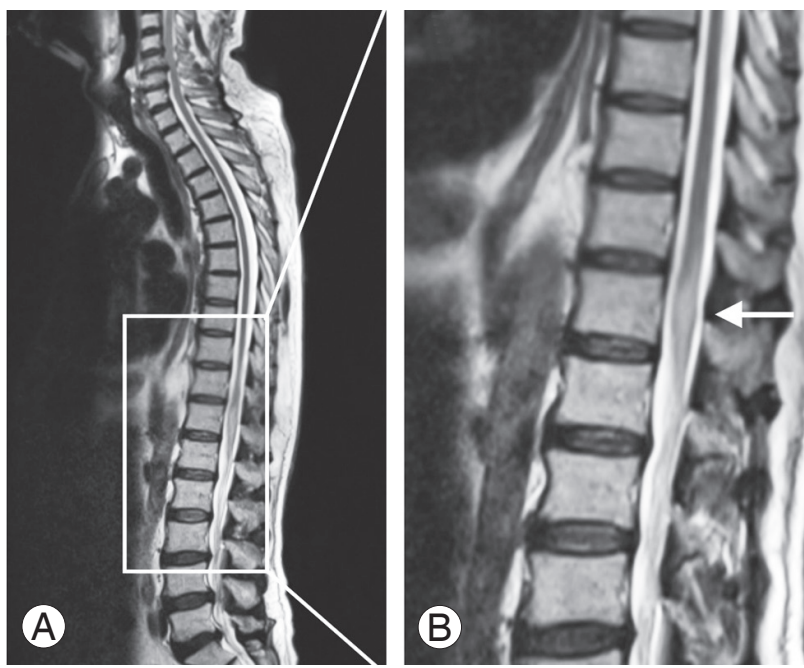

Fig. 4. A 39-year-old man presented with low back pain without any neurological symptoms. (A, B) T2-weighted sagittal screening of the whole spine revealed a hyperintense intramedullary at the conus medullaris (arrow). The lesion was confirmed as ependymoma.
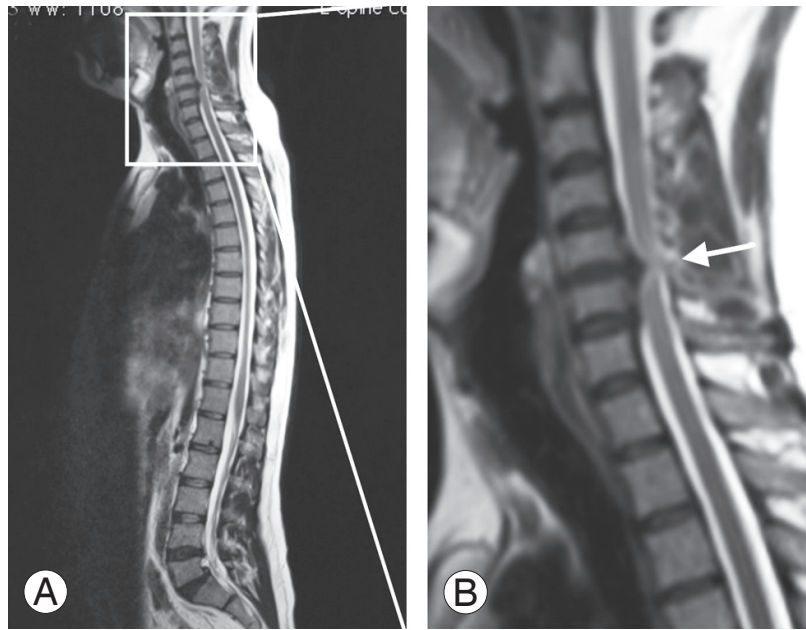

Fig. 5. A 46-year-old man presented with low back pain without any neurological symptoms. (A, B) T2-weighted sagittal screening of the whole spine revealed degenerative disease at the C5-6 intervertebral disc that was causing significant spinal cord compression with myelomalacic changes at this level (arrow). The lumbar spine was unremarkable except for bilateral spondylolysis at LV5 with associated anterolisthesis of LV5 over SV1.

(Fig. 4), with images obtained in multiple planes allowing the best definition of the lesion.

Another incidental finding we observed was myelomalacia in the cervicothoracic spine (Fig. 5). The onset of myelomalacia is usually so subtle that it is often overlooked. If detected in the early stages, steroids can play a role in reducing pain, cord edema, and spasticity. In rare cases, surgery may slow or stop further damage. Imaging

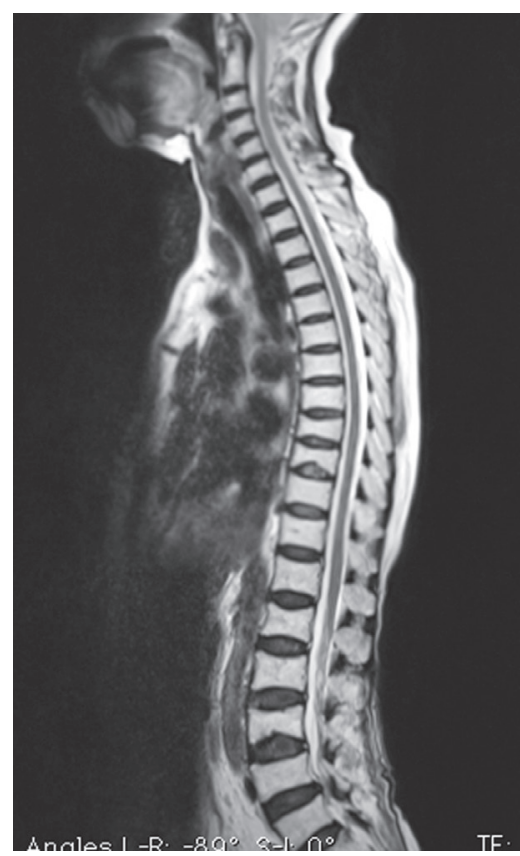

Fig. 6. Multiple incidentally detected dorsal vertebral fractures in a 54-year-old postmenopausal woman.

the entire spine using MRI allows early detection of these changes.

Vertebral fractures (Fig. 6) are the most common osteoporotic fracture; the lifetime probability of experiencing a clinical vertebral fracture (a painful vertebral fracture with a clinical diagnosis) is approximately $15 \%$ [10]. According to the literature, however, about two-thirds of patients with a vertebral fracture detected on imaging had received no prior diagnosis of this [11]. Undiagnosed and clinically diagnosed radiographic vertebral fractures have been associated with pain, physical disability, spinal deformity, and a decline in quality of life [12]. Because there are available drug treatments that can substantially reduce the risk of future fractures, it is important to identify people with existing vertebral fractures before the condition progresses further [13].

A limitation of our study was that the findings were not surgically confirmed (with the exception of the spaceoccupying lesions in the thoracic spine). The positive findings were diagnosed through MRI only. Because most of the findings were benign, no active surgical intervention was mandated in the majority of these cases.

\section{Conclusions}

Early detection of incidental lesions provides clinicians 
with an opportunity to offer early intervention to these patients. In our study, T2-weighted sagittal screening of whole spine detected incidental positive findings in approximately $26.7 \%$ of the patients undergoing lumbar MRI. Even though this requires additional scanning time of about 1.5 minutes (depending on the acquisition parameters) but the advantages gained from incorporating this sequence far outweigh this modest increase in examination time. We propose that sagittal screening of the whole spine should be included as a routine sequence when imaging the lumbosacral spine for suspected degenerative pathology of intervertebral discs.

\section{Conflict of Interest}

No potential conflict of interest relevant to this article was reported.

\section{ORCID}

Akhilesh Rao: https://orcid.org/0000-0002-1936-2939

\section{References}

1. Apazidis A, Ricart PA, Diefenbach CM, Spivak JM. The prevalence of transitional vertebrae in the lumbar spine. Spine J 2011;11:858-62.

2. Weyreuther M, Heyde CE, Weber U, Westphal M, Zierski J. MRI atlas orthopedics and neurosurgery the spine. Berlin: Springer-Verlag Berlin Heidelberg; 2007.

3. Konin GP, Walz DM. Lumbosacral transitional vertebrae: classification, imaging findings, and clinical relevance. AJNR Am J Neuroradiol 2010;31:1778-86.

4. Kim JT, Bahk JH, Sung J. Influence of age and sex on the position of the conus medullaris and Tuffier's line in adults. Anesthesiology 2003;99:1359-63.

5. Maus TP. Spine imaging, an issue of radiologic clinics of North America. London: Elsevier Health Sciences; 2012.

6. Pueschel SM, Scola FH. Atlantoaxial instability in individuals with Down syndrome: epidemiologic, radiographic, and clinical studies. Pediatrics 1987;80:555-60.

7. Neva MH, Hakkinen A, Makinen H, Hannonen P, Kauppi M, Sokka T. High prevalence of asymptomatic cervical spine subluxation in patients with rheumatoid arthritis waiting for orthopaedic surgery. Ann Rheum Dis 2006;65:884-8.

8. Yang SY, Boniello AJ, Poorman CE, Chang AL, Wang S, Passias PG. A review of the diagnosis and treatment of atlantoaxial dislocations. Global Spine J 2014;4:197-210.

9. Chamberlain MC, Tredway TL. Adult primary intradural spinal cord tumors: a review. Curr Neurol Neurosci Rep 2011;11:320-8.

10. Lauritzen JB, Schwarz P, Lund B, McNair P, Transbol I. Changing incidence and residual lifetime risk of common osteoporosis-related fractures. Osteoporos Int 1993;3:127-32.

11. Ross PD. Clinical consequences of vertebral fractures. Am J Med 1997;103:30S-42S.

12. Nevitt MC, Ettinger B, Black DM, et al. The association of radiographically detected vertebral fractures with back pain and function: a prospective study. Ann Intern Med 1998;128:793-800.

13. Meunier PJ, Delmas PD, Eastell R, et al. Diagnosis and management of osteoporosis in postmenopausal women: clinical guidelines: International Committee for Osteoporosis Clinical Guidelines. Clin Ther 1999;21:1025-44. 